\title{
A N-terminal truncated intracellular isoform of matrix metalloproteinase-2 impairs contractility of mouse myocardium
}

\author{
David H. Lovett ${ }^{1,2}$, Charles Chu ${ }^{1,2}$, Guanying Wang ${ }^{1,2}$, Mark B. Ratcliffe ${ }^{1,3,4}$ and Anthony J. Baker ${ }^{1,2 *}$ \\ ${ }^{1}$ Cardiology Division, San Francisco Veteran Affairs Medical Center, San Francisco, CA, USA \\ 2 Department of Medicine, University of California, San Francisco, San Francisco, CA, USA \\ ${ }^{3}$ Department of Surgery, University of California, San Francisco, San Francisco, CA, USA \\ 4 Joint UC Berkeley/UCSF Bioengineering Group, San Francisco, CA, USA
}

\section{Edited by:}

Jolanda Van Der Velden, VU University Medical Center,

Netherlands

\section{Reviewed by:}

Regis Lamberts, University of Otago, New Zealand

Lewis J. Watson, Duke University, USA

Eugenio Cingolani, Cedars Sinai Heart Institute, USA

*Correspondence: Anthony J. Baker, Cardiology Division (111C), San Francisco Veterans Affairs Medical Center, University of California, San Francisco, 4150 Clement St., San Francisco, CA 94121, USA e-mail: anthony.baker@ucsf.edu
The full-length isoform of matrixmetalloproteinase-2 (FL-MMP-2) plays a role in turnover of the cardiac extracellular matrix. FL-MMP-2 is also present intracellularly in association with sarcomeres and, in the setting of oxidative stress, cleaves myofilament proteins with resultant impaired contractility. Recently, a novel N-terminal truncated MMP-2 isoform (NTT-MMP-2) generated during oxidative stress was identified and shown to induce severe systolic failure; however, the injury mechanisms remained unclear. In this study, cardiac-specific NTT-MMP-2 transgenic mice were used to determine the physiological effects of NTT-MMP-2 on: force development of intact myocardium; the function of cardiac myofilaments in demembranated myocardium; and on intracellular $\mathrm{Ca}^{2+}$ transients in isolated myocytes. We related the contractile defects arising from NTT-MMP-2 expression to the known intracellular locations of NTT-MMP-2 determined using immunohistochemistry. Comparison was made with the pathophysiology arising from cardiac-specific FL-MMP-2 transgenic mice. Consistent with previous studies, FL-MMP-2 was localized to myofilaments, while NTT-MMP-2 was concentrated within subsarcolemmal mitochondria and to sites in register with the Z-line. NTT-MMP-2 expression caused a $50 \%$ reduction of force development by intact myocardium. However, NTT-MMP-2 expression did not reduce myofilament force development, consistent with the lack of NTT-MMP-2 localization to myofilaments. NTT-MMP-2 expression caused a $50 \%$ reduction in the amplitude of $\mathrm{Ca}^{2+}$ transients, indicating impaired activation.

Conclusions: Unlike FL-MMP-2, NTT-MMP-2 does not mediate myofilament damage. Instead, NTT-MMP-2 causes impaired myocyte activation, which may involve effects due to localization in mitochondria and/or to transverse tubules affecting $\mathrm{Ca}^{2+}$ transients. Thus, FL-MMP-2 and NTT-MMP-2 have discrete intracellular locations and mediate different intracellular damage to cardiac myocytes.

Keywords: contraction, heart failure, myofilament, $\mathrm{Ca}^{2+}$ transient, remodeling, MMP-2

\section{INTRODUCTION}

While the overall mortality rates for acute myocardial infarction have decreased in recent years due to the advent of more effective acute interventions, the incidence of dysfunctional postmyocardial ventricular remodeling continues to increase. The most serious complication of post-infarction remodeling is congestive heart failure, which has become one of the leading causes of death in ischemic cardiac disease. Recent experimental and clinical evidence has underscored a critical role for matrix metalloproteinase-2 (MMP-2) in ischemic cardiac disease, including post-infarction ventricular remodeling (AlfonsoJaume et al., 2006; Mukherjee et al., 2006; Bergman et al., 2007; Zhou et al., 2007; Nilsson et al., 2011; Lovett et al., 2012, 2013; Cogni et al., 2013). Our current gaps in knowledge are substantial and relate to an incomplete understanding of the multiple pathophysiologic roles of MMP-2 in post-infarction remodeling.

MMP-2 affects multiple factors involved in ischemic ventricular remodeling, inflammation and cardiomyocyte apoptosis. MMP-2 was originally investigated as the full-length secreted enzyme involved in the turnover of the extracellular matrix. However, recent studies have defined a pool of intracellular MMP2 arising from a fraction of full-length MMP-2 (FL-MMP-2) that escapes from the secretory pathway and, in the setting of redox stress, causes intracellular damage to the myofilaments in cardiomyocytes (Wang et al., 2002; Sawicki et al., 2005; Schulz, 2007; Lovett et al., 2012, 2013).

Under pathophysiological conditions, MMP-2 is known to be associated with cardiac dysfunction. To determine the role of MMP-2 in the absence of superimposed injury, we previously 
used cardiac-specific transgenic mice that expressed constitutively active FL- MMP-2 (Wang et al., 2006; Bergman et al., 2007). We reported that FL-MMP-2 expression resulted in a 50\% reduction of contractile force of electrically stimulated cardiac trabeculae. Furthermore, impaired force development was associated with a $50 \%$ reduction of myofilament force development assessed using demembranated myocardium exposed to activating solutions (Wang et al., 2006). These studies suggest that, in the absence of superimposed injury, FL-MMP-2 impairs myocardial contraction by damaging the myofilaments. Consistent with this, FL-MMP-2 localizes to the myofilaments (Bergman et al., 2007) and recent studies demonstrate that intracellular FL-MMP-2 cleaves several myofilament-associated proteins, including troponin I, titin, $\alpha$-actinin and myosin essential light chain (Ali et al., 2011).

We recently identified a second intracellular MMP-2 isoform, N-terminal truncated MMP-2 (NTT-MMP-2) that is generated by redox stress-mediated activation of an alternate promoter in the first intron of the MMP-2 gene (Lovett et al., 2012). Oxidative stress-mediated generation of NTT-MMP-2 may contribute to progressive cardiac dysfunction in the setting of ischemia (Lovett et al., 2012). Translation of the NTT-MMP-2 transcript is initiated at Methionine ${ }^{77}$, resulting in an $\mathrm{N}$-terminal truncated MMP-2 protein lacking the secretory sequence and the inhibitory prodomain. Thus, the NTT-MMP-2 protein remains intracellular and is enzymatically active. In-vitro cell fractionation studies localized the NTT-MMP-2 isoform to cytosolic and mitochondrial fractions (Lovett et al., 2012). Within the context of the intact heart, the NTT-MMP-2 isoform was primarily localized to mitochondria and also localized to perpendicular arrays across the long axis of individual cardiomyocytes (Lovett et al., 2013). In addition to activation of NTT-MMP-2, ischemic cardiac injury involves activation of multiple other factors. Therefore, for the current study, to focus only on the physiological effects mediated by NTT-MMP-2, we used transgenic expression of NTTMMP-2 in mouse hearts. Cardiac-specific transgenic expression of the NTT-MMP-2 isoform induced severe systolic failure; however, the injury mechanisms involved were unclear (Lovett et al., 2013). Therefore, the goal of this study was to investigate the mechanisms of NTT-MMP-2-mediated cardiac systolic failure. We report that the NTT-MMP-2 isoform directly impairs cardiomyocyte contractility by affecting calcium handling. Thus, the FL-MMP-2 and NTT-MMP-2 isoforms contribute differently to cardiac contractile dysfunction.

\section{MATERIALS AND METHODS TRANSGENIC MICE}

This institution is accredited by the American Association for the Accreditation of Laboratory Animal Care (Institutional PHS Assurance Number is A3476-01). The investigation was approved by the Animal Care and Use Subcommittee (IACUC) of the San Francisco Veterans Affairs Medical Center (protocol 09-053-03) and conformed to the Guide for the Care and Use of Laboratory Animals published by the National Institutes of Health (Revised 2011).

Cardiac-specific transgenic mice expressing the NTT-MMP-2 isoform were generated in the CD-1 background as previously described (Lovett et al., 2013). Four to five month old transgenic and littermate controls were used. Cardiac-specific transgenic mice expressing the FL-MMP-2 isoform were generated and characterized as reported (Bergman et al., 2007). Four to five month old transgenic mice and littermate controls were used.

\section{IMMUNOHISTOLOGICAL DETECTION OF MMP-2 ISOFORMS}

For localization of the c-myc-tagged FL-MMP-2 transgene, paraformaldehyde-fixed ventricular sections were incubated with murine monoclonal anti-c-myc (9E11, Abcam, Cambridge, MA), followed by the M.O.M. kit (Vector, Burlingame, CA) and development with VIP purple substrate (Vector) as previously described (Bergman et al., 2007).

Intracellular localization of the NTT-MMP-2-EGFP transgene was performed on deparaffinized sections following citrate antigen retrieval (Vector) using a 1:1000 dilution of rabbit polyclonal anti-GFP (Abcam, Ab6556) for $4 \mathrm{~h}$, followed by incubation with 1:2000 biotinylated goat-anti-rabbit IgG. Histochemical development with diaminobenzidine- $\mathrm{Ni}^{+2}$ (DAB- $\mathrm{Ni}^{+2}$, Vector) was restricted to $3 \mathrm{~min}$ to limit reaction product migration, followed by a hematoxylin counterstain. Images were digitally captured at high resolution (4000 dpi) using a Zeiss Nomarksy optics microscope. A dense focus of $\mathrm{DAB}-\mathrm{Ni}^{+2}$ reaction product in the transgenic image was sampled in Adobe Photoshop CS4. Foci with similar pixel densities were converted to a fluorescent pseudocolor using the Image Adjust/Change Color tool to enhance contrast with background $\mathrm{DAB}-\mathrm{Ni}^{+2}$ staining. The wild type control images were processed simultaneously in an identical fashion.

\section{CONTRACTION OF INTACT MYOCARDIUM}

Mice were deeply anesthetized with pentobarbital $(100 \mathrm{mg} / \mathrm{kg}$ i.p.) mixed with heparin $(100 \mathrm{U})$. Hearts were removed from anesthetized mice and flushed with modified Krebs-Henseleit, as previously described (Wang et al., 2006). A free-running trabecula was dissected from the right ventricle. For NTT-MMP-2 mice and wild type controls, the width, thickness and cross-sectional area of trabeculae were: $148 \pm 62 \mu \mathrm{m}, 79 \pm 16 \mu \mathrm{m}, 0.012 \pm$ $0.007 \mathrm{~mm}^{2}, n=4$; and $174 \pm 11 \mu \mathrm{m}, 123 \pm 18 \mu \mathrm{m}, 0.017 \pm$ $0.003 \mathrm{~mm}^{2}, n=4$; there was not a significant difference in these values between NTT-MMP-2 and wild type ( $P>0.05$, ANOVA). Trabeculae were placed in a muscle chamber and mounted to a force transducer, and sarcomere length set to $2.1 \mu \mathrm{m}$. Trabeculae were superfused with Krebs-Henseleit solution at $22^{\circ} \mathrm{C}$ and field stimulated $(0.5 \mathrm{~Hz}$ pacing frequency and voltage $1.5 \times$ threshold $)$ (Wang et al., 2006).

\section{ASSESSMENT OF MYOFILAMENT CONTRACTION}

After recording electrically stimulated contractions, these same trabeculae were demembranated using $1 \%$ Triton X-100, and in-vitro myofilament function was assessed using steady state contractions at various bath $\left[\mathrm{Ca}^{2+}\right]$ as previously described (Wang et al., 2006). The relationship between steady state force (F) and $\left[\mathrm{Ca}^{2+}\right]$ was fit to the Hill equation: $\mathrm{F}=\mathrm{F}_{\max } \times$ $\left[\mathrm{Ca}^{2+}\right]^{n_{\mathrm{H}}} /\left(\left[\mathrm{Ca}^{2+}\right]^{n_{\mathrm{H}}}+\mathrm{EC}_{50}{ }^{n_{\mathrm{H}}}\right)$, where $\mathrm{F}_{\max }$ is the maximum $\mathrm{Ca}^{2+}$-activated force, $\mathrm{EC}_{50}$ is the $\left[\mathrm{Ca}^{2+}\right]$ at which $\mathrm{F}$ is $50 \%$ of $\mathrm{F}_{\max }$, and $n_{\mathrm{H}}$ is Hill coefficient reflecting the slope of the $\mathrm{Ca}^{2+}$-force relationship at $\mathrm{EC}_{50}$. 


\section{MEASUREMENT OF $\mathrm{Ca}^{2+}$ TRANSIENTS}

Myocyte $\mathrm{Ca}^{2+}$ transients were recorded using from the fluorescence of Fura-2 as we recently described (Chu et al., 2013). Briefly, mice were deeply anesthetized with pentobarbital $(100 \mathrm{mg} / \mathrm{kg}$ i.p.) mixed with heparin (100 U). Hearts were rapidly removed and immersed in ice-cold arrest solution (in $\mathrm{mM}: \mathrm{NaCl} 120, \mathrm{KCl} 30, \mathrm{CaCl}_{2}$ 0.1). Myocytes were isolated by enzymatic digestion of the heart by retrograde perfusion of the aorta with collagenase solution (O'Connell et al., 2007). After collagenase treatment, the heart was placed in a "stop buffer" to halt enzymatic digestion (O'Connell et al., 2007). The heart was placed in $10 \mathrm{~mL}$ of stop buffer and gently teased with fine forceps followed by repeated pipetting to release the cells. The cell isolation buffers contained $10 \mathrm{mM} \mathrm{BDM}$ (2,3-Butanedione monoxime) to prevent cell contraction. Cells were used within $4 \mathrm{~h}$ of isolation. We studied 38 cells from 9 animals.

Myocytes were loaded with the $\mathrm{Ca}^{2+}$ indicator Fura-2 by exposure to $1 \mu \mathrm{M}$ Fura-2-AM for $20 \mathrm{~min}$. After washing, myocytes were equilibrated for $20 \mathrm{~min}$ to allow for de-esterification of the indicator and used within $1.5 \mathrm{~h}$. after completion of the loading protocol.

Myocytes were superfused in a small glass-floored chamber with Krebs Henseleit solution containing (in $\mathrm{mM}$ ): $\mathrm{NaCl}, 112$; $\mathrm{KCl}, 5 ; \mathrm{MgCl}_{2}, 1.2$; glucose, $10 ; \mathrm{NaHCO}_{3}, 24 ; \mathrm{Na}_{2} \mathrm{SO}_{4}, 1.2$; $\mathrm{NaH}_{2} \mathrm{PO}_{4}, 2.0$; and $\mathrm{CaCl}_{2}, 1$. The perfusate was oxygenated with $95 \% \mathrm{O}_{2} / 5 \% \mathrm{CO}_{2}$ to give a $\mathrm{pH}$ of 7.4 at $22^{\circ} \mathrm{C}$. The chamber was mounted on an inverted Nikon Diaphot microscope and cells visualized at $\mathrm{x} 40$ magnification. Cells were electrically stimulated with $4 \mathrm{~ms}$ square wave pulses, at a frequency of $0.5 \mathrm{~Hz}$. Cell contraction and Fura-2 fluorescence were monitored using an IonOptix Hyperswitch system (Milton, MA). Cell contraction was computed by monitoring changes in muscle sarcomere length measured from the myocyte striation spacing. Fura-2 fluorescence at an emission wavelength of $510 \mathrm{~nm}$ was measured, with the excitation alternated between 340 and $380 \mathrm{~nm}$ at a frequency of $240 \mathrm{~Hz}$. Background autofluorescence at each wavelength was subtracted before computing the Fura-2 fluorescence ratio.

\section{STATISTICAL ANALYSIS}

Data presented as mean \pm SE. Statistical comparison used unpaired Student's $t$-test, and Two-Way ANOVA with repeated measures followed by the Bonferroni's post-hoc test to compare between groups. The significance level was set at $P<0.05$.

\section{RESULTS}

\section{DISTINCT LOCALIZATION OF NTT-MMP-2 vs. FL-MMP-2}

Figure 1 shows immunohistochemical staining of ventricular myocardium from NTT-MMP-2 transgenic mice. Punctate staining for eGFP-tagged NTT-MMP-2 transgene is concentrated within subsarcolemmal mitochondria (Figure 1B) consistent with our previous report (Lovett et al., 2013). In addition, punctate staining for the NTT-MMP-2 transgene is localized to discrete clusters that are in register with the Z-line of the sarcomere (Figure 1C), which may reflect structures associated with transverse tubules. This is consistent with our previous finding of NTT-MMP-2 localized to perpendicular arrays across the long axis of individual cardiomyocytes (Lovett et al., 2013). Myocardium from wild type mice did not show specific localization of signal for NTT-MMP-2 (Figure 1A).

Figure 2 shows immunohistochemical staining of ventricular myocardium from FL-MMP-2 transgenic mice. Consistent with our previous report (Bergman et al., 2007), a prominent striated pattern is evident in the stained section consistent with a sarcomeric localization of the c-myc tagged FL-MMP-2 transgene (Figure 2B). Ventricular myocardium from wild type mice did not show significant staining for the FL-MMP-2 transgene (Figure 2A).

These findings confirm that the NTT-MMP-2 and FL-MMP-2 isoforms were localized to distinct intracellular compartments.

\section{TRANSGENIC NTT-MMP-2 EXPRESSION REDUCED MYOCARDIAL FORCE DEVELOPMENT}

Figure 3A shows electrically stimulated force development of myocardium from wild type and NTT-MMP-2 transgenic hearts. Extracellular bath $\left[\mathrm{Ca}^{2+}\right]$ was varied to assess force development over the full range of cardiac muscle activation. Figure $3 \mathrm{~A}$ shows considerably lower force development by intact myocardium
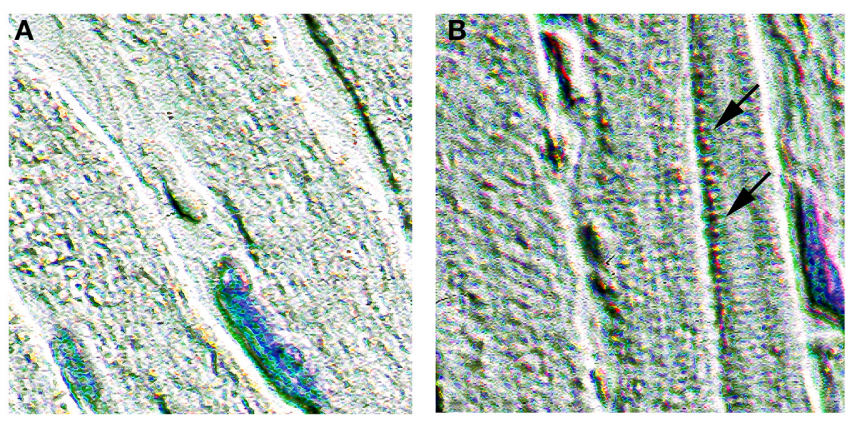

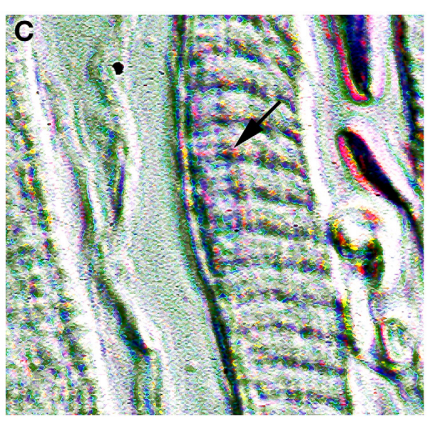

FIGURE 1 | Expression of NTT-MMP-2 in transgenic hearts.

Immunohistochemical staining for the eGFP epitope tag present on the NTT-MMP-2 transgene. Pseudo-color enhanced immunohistochemistry of wild type (A) and transgenic (B,C) ventricular sections $(400 \times$ original magnification). NTT-MMP-2 is present within mitochondria, particularly within subsarcolemmal mitochondria (indicated by arrows in $\mathbf{B}$ ) and localizes to clusters in register with the Z-line (arrow in C). No NTT-MMP-2 staining pattern was evident in wild type hearts. Intracellular NTT is much less abundant that the FL-MMP-2 isoform, therefore, pseudo-color enhancement was used to improve contrast and to visualize the subcellular localization. 


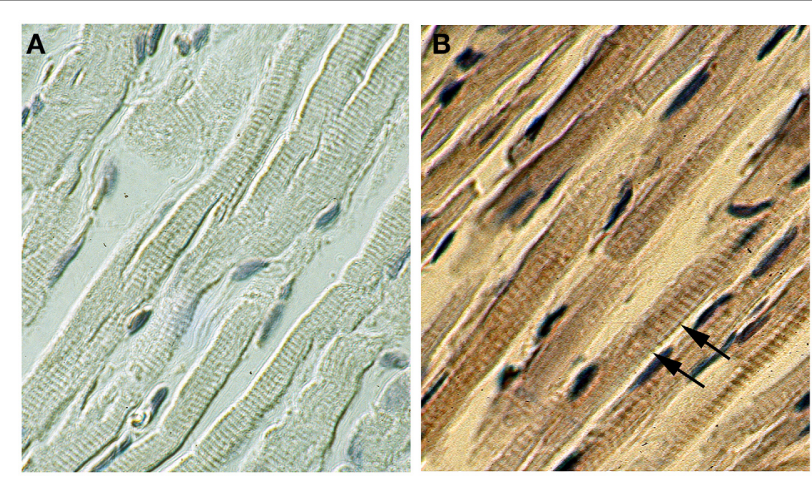

FIGURE 2 | Expression of FL-MMP-2 in transgenic hearts.

Immunohistochemical staining for the c-myc epitope tag of the FL-MMP-2 transgenic using peroxidase stain for wild type (A) and transgenic (B) ventricular sections (400x original magnification). The staining in a regular banding pattern (indicated by arrows in $\mathbf{B}$ ) is consistent with a sarcomeric localization. No staining was evident in wild type.

from NTT-MMP-2 transgenic mice compared to wild type mice, at the higher levels of activation. Diastolic force levels were low, with no difference in diastolic force level between NTT-MMP-2 transgenic vs. wild type myocardium $(0.79 \pm 0.25$ vs. $0.76 \pm 0.22$ $\left.\mathrm{mN} / \mathrm{mm}^{2}, P>0.05\right)$.

Thus, both transgenic NTT-MMP-2 expression, and transgenic FL-MMP-2 expression (Wang et al., 2006) cause similar reductions of force development by intact myocardium.

\section{TRANSGENIC NTT-MMP-2 EXPRESSION DID NOT AFFECT MYOFILAMENT FORCE DEVELOPMENT}

These same trabeculae were chemically skinned and exposed to activating solutions with various levels of free $\left[\mathrm{Ca}^{2+}\right]$ to measure myofilament force, separate from influences from the muscle activation system or muscle energy stores. Figure 3B shows that myofilament force development by trabeculae from NTT-MMP2 hearts was indistinguishable from that of trabeculae from wild type hearts. Furthermore, Hill parameters calculated by fitting myofilament force- $\mathrm{Ca}^{2+}$ relationships to the Hill equation were not different between NTT-MMP-2 and wild type (Figure 4).

The data from skinned myocardium suggests that the decreased force development of intact NTT-MMP-2 trabeculae (Figure 3A) did not involve intrinsically impaired myofilament function. This conclusion is consistent with the histology data showing that NTT-MMP-2 did not localize to the myofilaments (Figure 2). The absence of an effect of NTT-MMP-2 on myofilament force suggests that NTT-MMP-2 reduced the active force development of intact muscle by impairing other processes required for force generation such as impaired muscle activation.

These findings with NTT-MMP-2 expression differ considerably from those obtained with FL-MMP-2 expression, which was associated with markedly reduced myofilament force development (Wang et al., 2006). This suggests that there are distinctly different mechanisms involved in the reductions of myocardial force mediated by NTT-MMP expression vs. FL-MMP-2 expression. With FL-MMP-2 reducing myofilament contraction, but NTT-MMP-2 not affecting myofilament contraction.

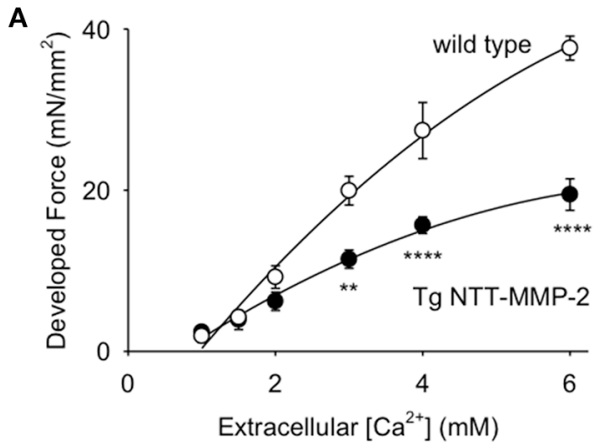

B

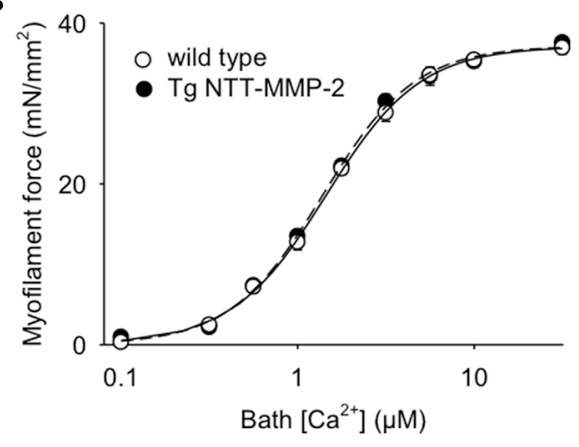

FIGURE 3 | (A) Relationship between developed force vs. bath [Ca ${ }^{2+}$ ] for electrically stimulated intact trabeculae. Trabeculae from transgenic (Tg) NTT-MMP-2 mice developed less force with raised bath $\left[\mathrm{Ca}^{2+}\right]$ compared to wild type controls ( $P<0.001$, ANOVA; mean $\pm \mathrm{SE}, n=4$ /group, $\left.{ }^{* *} P<0.01,{ }^{* * * *} P<0.0001\right)$. (B) Relationship between steady-state force development and $\left[\mathrm{Ca}^{2+}\right.$ ] for skinned trabeculae (error bars were less than the symbol size). There was no statistical difference in myofilament force development of trabeculae from wild type vs. Tg NTT-MMP-2 mice (Two-Way ANOVA), thus, expression of NTT-MMP-2 did not reduce myofilament force in skinned myocardium. Lines show fitting of the pooled data to the Hill equation.

\section{TRANSGENIC NTT-MMP-2 EXPRESSION REDUCED MYOCYTE Ca ${ }^{2+}$ TRANSIENTS}

We recorded $\mathrm{Ca}^{2+}$ transients in electrically stimulated single cardiac myocytes using the fluorescent $\mathrm{Ca}^{2+}$ indicator Fura-2. Figure 5 shows $\mathrm{Ca}^{2+}$ transients recorded from single myocytes from wild type or NTT-MMP-2 transgenic hearts. The myocyte $\mathrm{Ca}^{2+}$ transient amplitude was appreciably reduced by transgenic NTT-MMP-2 expression compared to wild type. The pooled data show that the amplitude of the $\mathrm{Ca}^{2+}$ transient was reduced approximately $50 \%$ in NTT-MMP-2 myocytes vs. wild type myocytes (Figure 6A). The reduced $\mathrm{Ca}^{2+}$ transient caused by NTT-MMP-2 expression was due to a reduced systolic $\mathrm{Ca}^{2+}$ level without appreciable effect on diastolic $\mathrm{Ca}^{2+}$ level. Consistent with a similar diastolic $\mathrm{Ca}^{2+}$ level, the diastolic sarcomere length was identical in NTT-MMP-2 expressing myocytes $(1.80 \pm 0.01 \mu \mathrm{m}$, $n=16)$ vs. wild type myocytes $(1.80 \pm 0.01 \mu \mathrm{m}, n=22)$. The pooled data show that for myocytes expressing NTT-MMP-2, the amplitude of the $\mathrm{Ca}^{2+}$ transient and contractions were reduced compared to myocytes from wild type hearts (Figure 6B).

NTT-MMP-2 expression did not have an appreciable effect on the dynamics of contraction and relaxation of cardiac myocytes. 
A

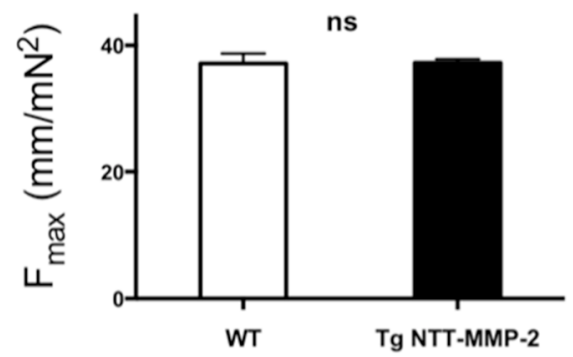

B

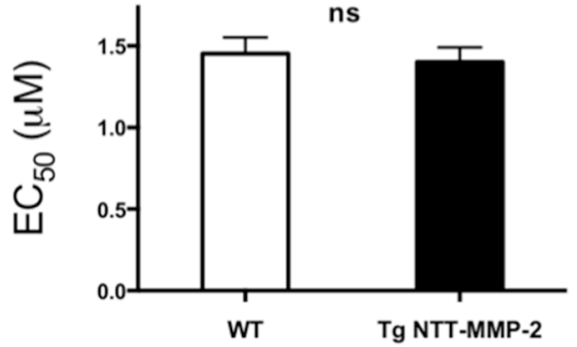

C

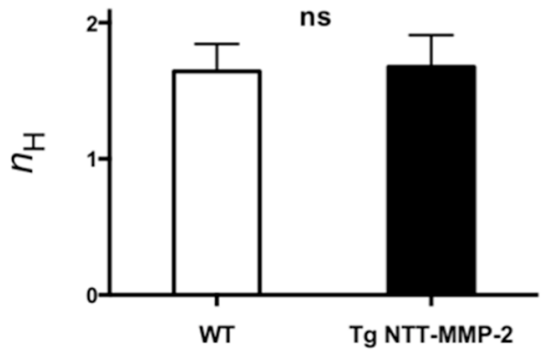

FIGURE 4 | Pooled data of Hill parameters derived from fitting the force- $\mathrm{Ca}^{2+}$ relationship for individual experiments to the Hill equation. (A) $F_{\max }$ (maximum $\mathrm{Ca}^{2+}$-activated force); (B) $\mathrm{Ca}^{2+}$ sensitivity $\mathrm{EC}_{50}\left(\left[\mathrm{Ca}^{2+}\right]\right.$ at $50 \% F_{\text {max }}$ ); (C) Hill coefficient $n_{H}$ (slope of $\mathrm{Ca}^{2+}$-force relationship at $\mathrm{EC}_{50}$ ). For all Hill parameters, no statistical differences were found between NTT-MMP-2 vs. wild type ( $P$ ns, Two-Way ANOVA; mean $\pm S E$, $n=4 /$ group)

For NTT-MMP-2 mice vs. wild type controls, the time to peak contraction and time to 50\% relaxation were: $92 \pm 8 \mathrm{~ms}$, and $90 \pm$ $7 \mathrm{~ms}, n=19$; vs. $95 \pm 5 \mathrm{~ms}$ and $100 \pm 10 \mathrm{~ms}, n=22$; there was not a significant difference in these values between NTT-MMP-2 and wild type $(P>0.05$, ANOVA).

In summary, the data show that unlike FL-MMP-2, NTTMMP-2 was not localized with the myofilaments, and did not cause impaired myofilament force development. NTT-MMP-2 caused impaired muscle activation, evidenced by decreased $\mathrm{Ca}^{2+}$ transients.

\section{DISCUSSION}

The major findings of this study were that transgenic expression of NTT-MMP-2 impaired myocardial contraction, and that impaired contraction did not involve decreased myofilament force. Instead, NTT-MMP-2 expression was associated with decreased calcium transients. These findings are in contrast to our previous findings that FL-MMP-2 expression resulted in decreased myocardial contraction that did involve
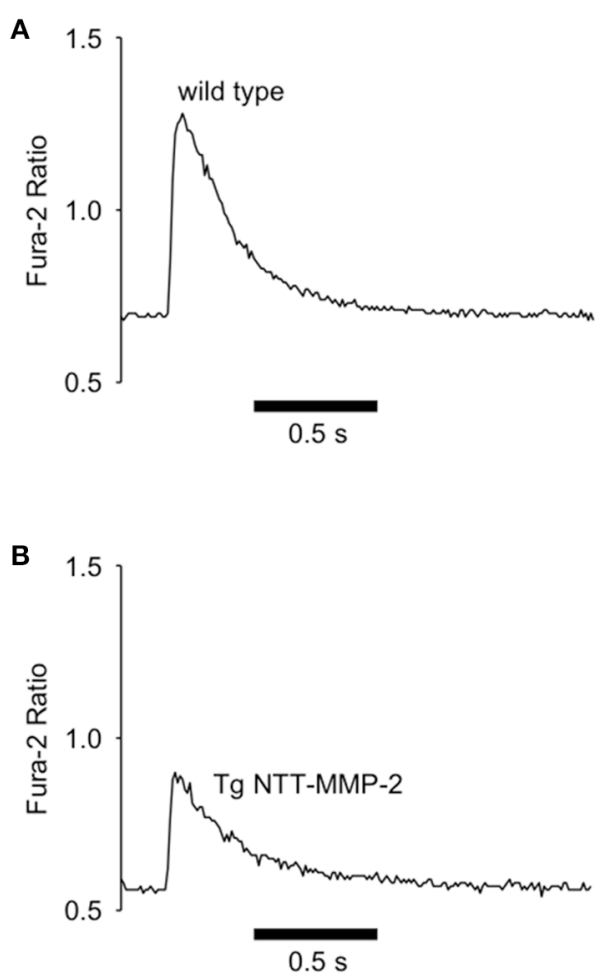

FIGURE 5 | Reduced $\mathrm{Ca}^{2+}$ transients with NTT-MMP-2 expression. Original records of Fura- $2 \mathrm{Ca}^{2+}$ transients recorded from electrically stimulated single cardiac myocytes. Compared to wild type controls (A) NTT-MMP-2 transgenic cells had reduced $\mathrm{Ca}^{2+}$ transient amplitude (B).

decreased myofilament force. Thus, the present study expands our understanding of the pathobiology of MMP-2 by demonstrating that two distinct isoforms of MMP-2 impair different intracellular processes.

Underscoring the disease relevance of MMP-2-mediated cardiac injury, studies in humans have implicated MMP-2 in LV dysfunction and dysfunctional remodeling after myocardial infarction (Nilsson et al., 2011; Cogni et al., 2013). Previous studies report decreased in-vivo function due to transgenic expression of FL-MMP-2 (Bergman et al., 2007) or NTT-MMP-2 (Lovett et al., 2013). Moreover, hemodynamic function measured in-vitro after ischemic injury showed reduced left ventricular developed pressure with transgenic expression of FL-MMP-2 (Zhou et al., 2007) or NTT-MMP-2 (Lovett et al., 2013).

FL-MMP-2 was confirmed to localize to the myofilaments (Bergman et al., 2007), consistent with a known effect of FLMMP-2 causing myofilament injury (Wang et al., 2006). In contrast, NTT-MMP-2 did not localize to myofilaments, and did not cause myofilament injury. Previously, NTT-MMP-2 was found to localize to mitochondria primarily within the subsarcolemmal space, and to perpendicular arrays across the long axis of individual cardiomyocytes (Lovett et al., 2013). The present study confirmed this and found that NTT-MMP-2 appeared within clusters along the Z-line.

These studies used transgenic NTT-MMP-2 mice at 4-5 months of age. At this age these mice have normal cardiac histology. Physiologic characterization of these mice with serial 

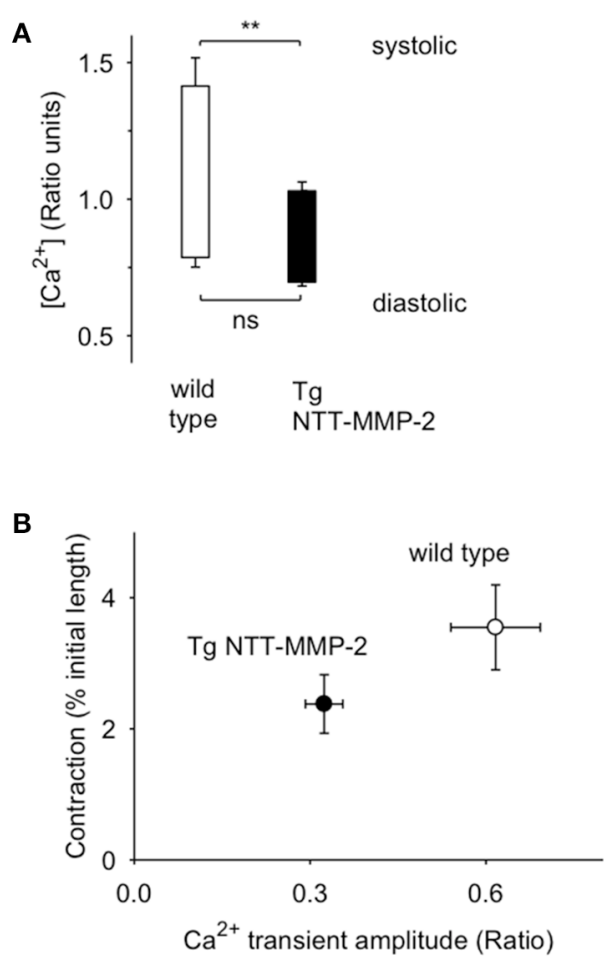

FIGURE 6 | Pooled data from cells from Tg NTT-MMP-2 mice and wild type. (A) Cells from Tg NTT-MMP-2 hearts had lower systolic $\mathrm{Ca}^{2+}$ levels than cells from wild type $(* * P<0.01)$. (B) Pooled data (mean \pm SE) for contraction amplitude vs. $\mathrm{Ca}^{2+}$ transient amplitude for cells from $\mathrm{Tg}$ NTT-MMP-2 hearts and wild type $[n=16.22$ cells/group from wild type mice $(n=5)$ and NTT-MMP-2 expressing mice $(n=4)]$. Cells from Tg NTT-MMP-2 hearts had smaller $\mathrm{Ca}^{2+}$ transients and contractions compared to wild type. There was a significant linear relation between contraction vs. $\mathrm{Ca}^{2+}$ transient amplitude $(P<0.001)$.

echocardiography revealed normal functional parameters other than a small decrease in left ventricular ejection fraction (Lovett et al., 2013). Thus, the impaired contractile function defined in the current study precedes the development of severe systolic dysfunction and is not a consequence thereof.

FL-MMP-2 is known to be involved in regulation of the cardiac extracellular matrix (Spinale, 2007), and to contribute to dysfunctional extracellular remodeling in the setting of myocardial infarction (Hayashidani et al., 2003). However, recent studies have recognized that FL-MMP-2 is also an intracellular protease (Schulz, 2007), with almost $40 \%$ of newly synthesized FL-MMP2 retained in the cytosol due to an inefficient secretory signal (Ali et al., 2012). In the setting of oxidative stress, intracellular FL-MMP-2 is activated by release of the inhibitory prodomain, leading to FL-MMP-2-mediated cleavage of sarcomeric proteins with resultant contractile dysfunction (Schulz, 2007).

NTT-MMP-2 is a novel intracellular MMP-2 isoform generated by oxidative stress-mediated activation of an alternate promoter in the first intron of the MMP-2 gene (Lovett et al., 2012, 2013). NTT-MMP-2 lacks the secretory peptide and the inhibitory prodomain and thus is enzymatically active and acts exclusively on intracellular targets. Like FL-MMP-2, cardiac-specific NTT-MMP-2 transgene expression also leads to systolic failure (Lovett et al., 2013). But the mechanisms involved have been unclear.

In contrast to FL-MMP-2, which localizes to the myofilaments, NTT-MMP-2 is not localized to the myofilaments. The absence of NTT-MMP-2 on the myofilaments is consistent with our finding that myofilament force was not impaired in NTT-MMP-2 transgenic hearts. Intact myocardium from hearts with transgenic expression of NTT-MMP-2 had a 50\% reduction in electrically stimulated myocardial force. Therefore, the reduced force of intact myocardium was not due to a reduction of intrinsic myofilament force. Instead, we found that NTT-MMP-2 was localized to subsarcolemmal mitochondria and to clusters that were in register with the Z-line of the sarcomere. NTT-MMP-2 localized to the $\mathrm{Z}$-line could reflect association with the transverse tubule (t-tubule), which is critically involved in excitation-contraction coupling. Disruptions of t-tubular structure and function have been implicated with impaired myocyte activation in heart failure (Cannell et al., 2006; Orchard and Brette, 2008). NTT-MMP2 effects on the t-tubule might be consistent with our finding that NTT-MMP-2 expression substantially impaired the amplitude of the $\mathrm{Ca}^{2+}$ transient in isolated myocytes. Impaired muscle activation with decreased $\mathrm{Ca}^{2+}$ transients may play a role in the reduced myocardial contraction caused by NTT-MMP-2 expression.

A prominent ultrastructural feature of the NTT-MMP-2 transgenic mice was the presence of swollen mitochondria with loss of organized cristae (Lovett et al., 2013). These morphologic features are characteristic of the opening of the mitochondrial permeability transition pore (MTP) (Buki et al., 2000). MTP opening is associated with enhanced oxidative stress due to uncoupling of the mitochondrial electron transport chain (Halestrap and Pasdois, 2009). Cardiac oxidant stress has multiple deleterious effects on calcium and sodium handling, which may also contribute to impaired contraction and $\mathrm{Ca}^{2+}$ transients associated with NTT-MMP-2 expression (Sag et al., 2013).

\section{LIMITATIONS}

We studied contracting cells and trabeculae, and demembranated trabeculae at $22^{\circ} \mathrm{C}$. While demembranated trabeculae are more stable for study at low temperature, nevertheless, temperature is known to affect contraction and relaxation properties (Janssen et al., 2002). Although wild type and transgenic groups were studied under the same conditions, caution is needed for extrapolating from the results of this study to the intact heart. Furthermore, to explore the full range of muscle activation, we used high bath $\mathrm{Ca}^{2+}$ levels in both intact and skinned myocardium. High $\mathrm{Ca}^{2+}$ levels and maximal levels of activation are not achieved in-vivo and therefore, caution is needed for extrapolating from these experimental conditions. To understand the basis of decreased $\mathrm{Ca}^{2+}$ transients with transgenic NTT-MMP-2 expression, mechanistic studies are needed to investigate the roles of mitochondria and $\mathrm{Ca}^{2+}$-handling systems that might be affected by NTT-MMP-2. Finally, as NTT-MMP-2 is activated by redox stress, greater understanding of the effect of NTT-MMP-2 expression in the context of redox stress is needed. 


\section{CONCLUSION}

Two discrete MMP-2 isoforms have distinctive pathophysiological mechanisms that impair cardiomyocyte contractility. FL-MMP-2 was previously reported to injure the myofilaments, in contrast, the current study found NTT-MMP-2 does not injure the myofilaments but impairs muscle activation involving reduced $\mathrm{Ca}^{2+}$ transients.

\section{ACKNOWLEDGMENTS}

This work was supported by Department of Veterans Affairs Merit Review Awards I01BX000593 (David H. Lovett), and I01BX000740 (Anthony J. Baker), National Heart, Lung and Blood Institute Grants R01-HL-84431 and R01-HL-63348 (Mark B. Ratcliffe), and an American Heart Association Grant-in-Aid 10GRNT3720074 (Anthony J. Baker). We thank Paul Wang, and Kevin V. Thai for expert technical assistance.

\section{REFERENCES}

Alfonso-Jaume, M. A., Bergman, M. R., Mahimkar, R., Cheng, S., Jin, Z. Q., Karliner, J. S., et al. (2006). Cardiac ischemia-reperfusion injury induces matrix metalloproteinase-2 expression through the AP-1 components FosB and JunB. Am. J. Physiol. Heart Circ. Physiol. 291, H1838-H1846. doi: 10.1152/ajpheart.00026.2006

Ali, M. A., Chow, A. K., Kandasamy, A. D., Fan, X., West, L. J., Crawford, B. D., et al. (2012). Mechanisms of cytosolic targeting of matrix metalloproteinase-2. J. Cell. Physiol. 227, 3397-3404. doi: 10.1002/jcp.24040

Ali, M. A., Fan, X., and Schulz, R. (2011). Cardiac sarcomeric proteins: novel intracellular targets of matrix metalloproteinase-2 in heart disease. Trends Cardiovasc. Med. 21, 112-118. doi: 10.1016/j.tcm.2012.03.008

Bergman, M. R., Teerlink, J. R., Mahimkar, R., Li, L., Zhu, B. Q., Nguyen, A., et al. (2007). Cardiac matrix metalloproteinase-2 expression independently induces marked ventricular remodeling and systolic dysfunction. Am. J. Physiol. Heart Circ. Physiol. 292, H1847-H1860. doi: 10.1152/ajpheart.00434.2006

Buki, A., Okonkwo, D. O., Wang, K. K., and Povlishock, J. T. (2000). Cytochrome c release and caspase activation in traumatic axonal injury. J. Neurosci. 20, 2825-2834.

Cannell, M. B., Crossman, D. J., and Soeller, C. (2006). Effect of changes in action potential spike configuration, junctional sarcoplasmic reticulum micro-architecture and altered t-tubule structure in human heart failure. J. Muscle Res. Cell Motil. 27, 297-306. doi: 10.1007/s10974-0069089-y

Chu, C., Thai, K., Park, K. W., Wang, P., Makwana, O., Lovett, D. H., et al. (2013). Intraventricular and interventricular cellular heterogeneity of inotropic responses to alpha(1)-adrenergic stimulation. Am. J. Physiol. Heart Circ. Physiol. 304, H946-H953. doi: 10.1152/ajpheart.00822.2012

Cogni, A. L., Farah, E., Minicucci, M. F., Azevedo, P. S., Okoshi, K., Matsubara, B. B., et al. (2013). Metalloproteinases-2 and -9 predict left ventricular remodeling after myocardial infarction. Arq. Bras. Cardiol. 100, 315-321. doi: 10.5935/abc. 20130049

Halestrap, A. P., and Pasdois, P. (2009). The role of the mitochondrial permeability transition pore in heart disease. Biochim. Biophys. Acta. 1787, 1402-1415. doi: 10.1016/j.bbabio.2008.12.017

Hayashidani, S., Tsutsui, H., Ikeuchi, M., Shiomi, T., Matsusaka, H., Kubota, T., et al. (2003). Targeted deletion of MMP-2 attenuates early LV rupture and late remodeling after experimental myocardial infarction. Am. J. Physiol. Heart Circ. Physiol. 285, H1229-H1235. doi: 10.1152/ajpheart.00207.2003

Janssen, P. M., Stull, L. B., and Marban, E. (2002). Myofilament properties comprise the rate-limiting step for cardiac relaxation at body temperature in the rat. Am. J. Physiol. Heart Circ. Physiol. 282, H499-H507. doi: 10.1152/ajpheart.00595. 2001

Lovett, D. H., Mahimkar, R., Raffai, R. L., Cape, L., Maklashina, E., Cecchini, G., et al. (2012). A novel intracellular isoform of matrix metalloproteinase-2 induced by oxidative stress activates innate immunity. PLOS ONE 7:e34177. doi: 10.1371/journal.pone.0034177

Lovett, D. H., Mahimkar, R., Raffai, R. L., Cape, L., Zhu, B. Q., Jin, Z. Q., et al. (2013). N-terminal truncated intracellular matrix metalloproteinase-2 induces cardiomyocyte hypertrophy, inflammation and systolic heart failure. PLoS ONE 8:e68154. doi: 10.1371/journal.pone.0068154

Mukherjee, R., Mingoia, J. T., Bruce, J. A., Austin, J. S., Stroud, R. E., Escobar, G. P., et al. (2006). Selective spatiotemporal induction of matrix metalloproteinase-2 and matrix metalloproteinase- 9 transcription after myocardial infarction. Am. J. Physiol. Heart Circ. Physiol. 291, H2216-H2228. doi: 10.1152/ajpheart.01343. 2005

Nilsson, L., Hallen, J., Atar, D., Jonasson, L., and Swahn, E. (2011). Early measurements of plasma matrix metalloproteinase-2 predict infarct size and ventricular dysfunction in ST-elevation myocardial infarction. Heart 98, 31-36. doi: 10.1136/heartjnl-2011-300079

O'Connell, T. D., Rodrigo, M. C., and Simpson, P. C. (2007). Isolation and culture of adult mouse cardiac myocytes. Methods Mol. Biol. 357, 271-296. doi: 10.1385/ 1-59745-214-9:271

Orchard, C., and Brette, F. (2008). t-Tubules and sarcoplasmic reticulum function in cardiac ventricular myocytes. Cardiovasc. Res. 77, 237-244. doi: 10.1093/cvr/ crm002

Sag, C. M., Wagner, S., and Maier, L. S. (2013). Role of oxidants on calcium and sodium movement in healthy and diseased cardiac myocytes. Free Radic. Biol. Med. 63, 338-349. doi: 10.1016/j.freeradbiomed.2013.05.035

Sawicki, G., Leon, H., Sawicka, J., Sariahmetoglu, M., Schulze, C. J., Scott, P. G., et al. (2005). Degradation of myosin light chain in isolated rat hearts subjected to ischemia-reperfusion injury: a new intracellular target for matrix metalloproteinase-2. Circulation 112, 544-552. doi: 10.1161/ CIRCULATIONAHA.104.531616

Schulz, R. (2007). Intracellular targets of matrix metalloproteinase-2 in cardiac disease: rationale and therapeutic approaches. Annu. Rev. Pharmacol. Toxicol. 47, 211-242. doi: 10.1146/annurev.pharmtox.47.120505.105230

Spinale, F. G. (2007). Myocardial matrix remodeling and the matrix metalloproteinases: influence on cardiac form and function. Physiol. Rev. 87, 1285-1342. doi: 10.1152/physrev.00012.2007

Wang, G. Y., Bergman, M. R., Nguyen, A. P., Turcato, S., Swigart, P. M., Rodrigo, M. C., et al. (2006). Cardiac transgenic matrix metalloproteinase-2 expression directly induces impaired contractility. Cardiovasc. Res. 69, 688-696. doi: 10.1016/j.cardiores.2005.08.023

Wang, W., Schulze, C. J., Suarez-Pinzon, W. L., Dyck, J. R., Sawicki, G., and Schulz, R. (2002). Intracellular action of matrix metalloproteinase-2 accounts for acute myocardial ischemia and reperfusion injury. Circulation 106, 1543-1549. doi: 10.1161/01.CIR.0000028818.33488.7B

Zhou, H. Z., Ma, X., Gray, M. O., Zhu, B. Q., Nguyen, A. P., Baker, A. J., et al. (2007). Transgenic MMP-2 expression induces latent cardiac mitochondrial dysfunction. Biochem. Biophys. Res. Commun. 358, 189-195. doi: 10.1016/j. bbrc.2007.04.094

Conflict of Interest Statement: The authors declare that the research was conducted in the absence of any commercial or financial relationships that could be construed as a potential conflict of interest.

Received: 27 June 2014; accepted: 03 September 2014; published online: 25 September 2014.

Citation: Lovett DH, Chu C, Wang G, Ratcliffe MB and Baker AJ (2014) A N-terminal truncated intracellular isoform of matrix metalloproteinase-2 impairs contractility of mouse myocardium. Front. Physiol. 5:363. doi: 10.3389/fphys.2014.00363

This article was submitted to Striated Muscle Physiology, a section of the journal Frontiers in Physiology.

Copyright (C) 2014 Lovett, Chu, Wang, Ratcliffe and Baker. This is an open-access article distributed under the terms of the Creative Commons Attribution License (CC BY). The use, distribution or reproduction in other forums is permitted, provided the original author(s) or licensor are credited and that the original publication in this journal is cited, in accordance with accepted academic practice. No use, distribution or reproduction is permitted which does not comply with these terms. 